\title{
Deposition of Ibuprofen Crystals on Hydroxypropyl Cellulose/Polyacrylamide Gel: Experimental and Mathematic Modeling Releasing
}

\author{
Claudia Alicia Castillo-Miranda, ${ }^{1}$ Ana Beatriz Morales-Cepeda, ${ }^{1}$ \\ Carlos Fernando Castro-Guerrero, ${ }^{2}$ Homero Salas-Papayanopolos, ${ }^{1}$ \\ Hugo Alberto Velasco-Ocejo, ${ }^{1}$ Jorge Alberto González-Sánchez, ${ }^{3}$ \\ Ulises Paramo-Garcia, ${ }^{1}$ and Hugo De Alva-Salazar ${ }^{1}$ \\ ${ }^{1}$ División de Estudios de Posgrado e Investigación, Instituto Tecnológico de Ciudad Madero, Juventino Rosas y Jesús Urueta s/n, \\ Colonia Los Mangos, 89318 Ciudad Madero, TAMPS, Mexico \\ ${ }^{2}$ CONACyT-Instituto de Investigaciones Eléctricas, Reforma 113, Colonia Palmira, Cuernavaca 62490, MOR, Mexico \\ ${ }^{3}$ Departamento de Ciencias Básicas del Instituto Tecnológico de Ciudad Madero, Juventino Rosas y Jesús Urueta s/n, \\ Colonia Los Mangos, 89318 Ciudad Madero, TAMPS, Mexico
}

Correspondence should be addressed to Ana Beatriz Morales-Cepeda; abmoralesc@itcm.edu.mx

Received 12 May 2016; Revised 28 August 2016; Accepted 6 September 2016

Academic Editor: Ming-Guo Ma

Copyright (C) 2016 Claudia Alicia Castillo-Miranda et al. This is an open access article distributed under the Creative Commons Attribution License, which permits unrestricted use, distribution, and reproduction in any medium, provided the original work is properly cited.

\begin{abstract}
The crystallization of nonsteroidal anti-inflammatory drug [2-(4-isobutyl-phenyl) propionic acid] ibuprofen (IBP) on a hydroxypropyl cellulose (HPC) and polyacrylamide (PAAm) gel was studied as well as the release kinetics of the drug. The IBP was crystallized on the gel surface of HPC/PAAm. It had a prismatic shape and the growth was made in an aqueous medium; the crystallinity grade of the gels HPC/PAAm and HPC/PAAm-IBU increased to $68 \%$ and to 58\%, respectively. The release of IBP is performed by two means: by a non-Fickian diffusion process and by relaxation of the chains of the gel; without regard to temperature and the diffusion media, this correlates with the lower critical solution temperature (LCST) of the proposed gel. This polymer matrix provides an option for releasing nonsteroidal anti-inflammatory drugs in a temperature range of $35-39^{\circ} \mathrm{C}$. Korsmeyer and Peppas mathematical model was simulated for data releases, statistically significant at $95 \%$ confidence level.
\end{abstract}

\section{Introduction}

Gels represent a major group of biomaterials; they are considered intelligent systems, as they have a swelling response depending upon selective environmental conditions such as $\mathrm{pH}$, temperature, ionic strength, and electric and magnetic field $[1,2]$. Within the gels we find hydrogels, which are synthesized using water as reacting medium. To achieve selective medium, responsive hydrogels have been synthesized from various homopolymers and copolymers, with recent wider applicability, as monomers combine to provide good mechanical properties, along with other monomers that give gels a hydrophilic material nature, as well as a selective response to environmental conditions.

In recent years, there has been an increased interest in hydrogels, and they have been extensively studied as drug delivery systems that allow the release of the right amount of the active ingredient, at the appropriate time and at specific sites within the body. Previous studies of the HPC/PAAm gels have been performed; the phase behavior has been studied, and it was found that the LCST and UCST (upper critical solution temperature) depend on the amount of HPC. The gel described in this paper has a LCST of $38.3^{\circ} \mathrm{C}$ and an UCST of $29.1^{\circ} \mathrm{C}[3]$. 
Several authors reported the simulation of drug releases from delivery system using hydroxypropyl methylcellulose (HPMC) [4] to elucidate the mass transport and possibility to predict the effect of design parameters tablets.

This research demonstrates that ibuprofen is crystallized on a HPC/PAAm gel and then released. Crystallization is due to the ability of IBP to form the crystals on diamond shape and to stack on the gel due to the solvent and to the LCST and UCST of the gels. The nonsteroidal anti-inflammatory drug (NSAID) release kinetics was studied in two media, buffer saline and ethanol-water system. The mathematical models were used to find a correlation with experimental data.

\section{Experimental}

2.1. Materials. In this paper we used hydroxypropyl cellulose (HPC) with average molecular weight (Mw) of $\sim 80,000 \mathrm{~g} /$ mol, acrylamide (AAm, purity 97\%), methylenebisacrylamide (MBAm, purity 99\%), tetramethylethylenediamine (TEMED, purity 99\%), ibuprofen (IBP), ammonium persulfate (APS, purity 98\%), and divinyl sulfone (DVS, purity 97\%), all of which were purchased from Sigma-Aldrich. Deionized (DI) water and phosphate buffered solution (PBS) at $\mathrm{pH} 7.38$ were supplied by Hycel.

2.2. Synthesis of Hydrogels. The synthesis of HPC/PAAm hydrogels was synthesized according to the method of Castro et al. [3] at a ratio of 25/75 wt $\%$. The reaction was carried out in a four-necked flask with a temperature control at $40 \pm 1^{\circ} \mathrm{C}$ and an inert nitrogen atmosphere. The solution consisted of $90 \%$ deionized water and $10 \%$ of reagents in the desired amount to work. At the beginning, $1 \mathrm{~g}$ of HPC was diluted in $20 \mathrm{~mL}$ of DI water, and the mixture was allowed to stir at room temperature for about 15 hours to achieve a homogeneous solution. Then, the reactor was purged with nitrogen and $3 \mathrm{~g}$ of AAm was added. Thus, $0.06 \mathrm{~g}$ of APS was dissolved with $0.003 \mathrm{~g}$ of MBAm in a vial containing $8 \mathrm{~mL}$ of DI water, and in another vial containing the same amount of water $0.06 \mathrm{~g}$ of TEMED was dissolved; both vials were stirred for 20 minutes. Once well dissolved, the content of first vial was injected in the reactor, then $0.3 \mathrm{~mL}$ of DVS was added, and finally the second vial was injected in the reactor. The polymerization was done for 1 hour at $40^{\circ} \mathrm{C}$ in an inert atmosphere and with constant stirring at $\mathrm{pH}$ 7. After the reaction, the solution was poured in a petri dish, and it was allowed to dry at $40^{\circ} \mathrm{C}$ in an oven with vacuum for one week. Once dried, the resulting films were washed with DI water in order to remove the nonreacted substances and then they were left to dry again.

2.3. Deposition of IBP. The deposition of IBP on the gel was carried out according to the method of Risbud et al. [5]. The NSAID was loaded into the samples by inserting them into an aqueous solution of the drug at a concentration of $5 \mathrm{mg} / \mathrm{mL}$ of IBP at room temperature for 48 hours. After this time, the excess of solution was removed from the gels, then frozen at $-10^{\circ} \mathrm{C}$ for 48 hours, and finally allowed to stand at room temperature for constant weight.
2.4. Drug Release. Once the samples were loaded with the drug, we proceeded to perform the release studies. These studies were done with the dried gel after the incorporation of the NSAID. All of the release experiments were carried out in a heating bath at a controlled temperature $(35,37$, and $39^{\circ} \mathrm{C}$ ) with electromagnetic vibrations ( 80 cycles/min) using as release means $\mathrm{PBS}$ at $\mathrm{pH} 7.4$ and ethanol-water solution $(50: 50)$ at $\mathrm{pH}$. The release kinetics was measured by taking samples at different intervals of time and determining the drug concentration by UV spectroscopy at wavelengths of $261 \mathrm{~nm}$ for samples released in PBS and $255 \mathrm{~nm}$ in samples released from ethanol-water solution using a UV-Vis spectrophotometer, Perkin-Elmer (model Lambda 10).

2.5. Kinetic Study of the Release of IBP. Release profiles (concentration of drug released versus time) were calculated using absorbance data. The nature of NSAID diffusion from the gels was determined to indicate what model it fits.

Mathematical models proposed by Higuchi [6] and Korsmeyer and Peppas [7] are among the most widely used to analyze and describe the mechanism by which the release process occurs. Higuchi proposed a mathematical model widely used to describe the empirical process of the drug delivery, which complies with Fick's law and is represented as follows:

$$
\frac{M_{t}}{M_{\infty}}=k t^{1 / 2},
$$

where $M_{t} / M_{\infty}$ is the released fraction of IBP in a time interval $t$ and $k$ is the constant release rate.

The mathematical model proposed by Korsmeyer and Peppas is linear for values equal to $M_{t} / M_{\infty}<0.6$. This model attempts to explain release mechanisms where erosion and/or dissolution of the matrix occurs and is a generalized form of Higuchi equation (1) [8], which is expressed as

$$
\frac{M_{t}}{M_{\infty}}=k t^{n},
$$

where $k$ is the release rate constant which incorporates structural and geometric features of the delivery system and $n$ is an exponent that indicates the mechanism by which drug release occurs. The exponent value $n$ provides information on drug's kinetics release; so if $n$ is equal to 0.5 , the drug release occurs through a diffusion phenomenon, Fickian (Higuchi mathematical model) type; if the value of $n$ falls between 0.5 and 1 , it indicates that the drug release is caused by a non-Fickian mechanism or anomalous diffusion, and when $n$ equals 1 , the mechanism of drug release depends on the process of relaxation of the polymer chains [1].

Equation (2) described the first $60 \%$ of the release behavior of hypothetical distribution. Later, Korsmeyer and Peppas [7] described an empirical equation, for three Fickian diffusional cases. The case II of the Peppas equations for Fickian and anomalous release was used in this study:

$$
\frac{M_{t}}{M_{\infty}}=\frac{2 k_{0}}{C_{0} l} t .
$$


2.6. Characterization of Gels. The samples were characterized using Fourier Transform Infrared (FTIR) spectroscopy in a Perkin-Elmer device (model Spectrum One) in attenuated total reflectance (ATR) mode using the frequency range $4000-600 \mathrm{~cm}^{-1}$. Thermal properties were carried out using Differential Scanning Calorimetry (DSC) in a Perkin-Elmer device model Pyris 1 . The sample was heated from $0^{\circ} \mathrm{C}$ to $200^{\circ} \mathrm{C}$ at a rate of $10^{\circ} \mathrm{C} / \mathrm{min}$, under a nitrogen atmosphere. Micrographs of the samples were prepared in a Scanning Electronic Microscope (SEM) JEOL model JSM-5900 using a size of sample $1 \mathrm{~cm}^{2}$; the gels were sputtered with a gold layer. The samples were analyzed with energy dispersive Xray spectroscopy (EDX), using the EDX instrumentation attached to the SEM, with a Bruker Analyzer operating at $133 \mathrm{eV}$. X-ray diffraction of the samples was performed with a Bruker AXS D8 Advance diffractometer using $\mathrm{CuK} \alpha$ radiation; the samples were cut into squares with sides of $0.5 \mathrm{~cm}$.

Degree of crystallinity was calculated according to

$$
X_{C}=\frac{I_{\text {Crystalline }}}{I_{\text {Crystalline }}+I_{\text {Amorphous }}} * 100 \text {. }
$$

The degree of crystallinity of the gels was calculated by Peak Height method. For cellulosic materials, the apparent crystallinity is calculated from the height ratio between the intensity of the crystalline peak $\left(22-24^{\circ}\right.$ in $\left.2 \theta\right)$ and the intensity of the noncrystalline material $\left(18^{\circ}\right.$ in $\left.2 \theta\right)$.

\section{Results and Discussion}

3.1. Infrared Spectroscopy. The infrared spectrum of the synthesized xerogel HPC/PAAm is shown in Figure 1(c). At $3338 \mathrm{~cm}^{-1}$, a broad peak attributable to the strong symmetric stretching $\mathrm{NH}$ of the PAAm can be seen and the peak at $3182 \mathrm{~cm}^{-1}$ is another one of high intensity corresponding to the stretch $\mathrm{OH}$ in the HPC [8]. Moreover, the peak at $2929 \mathrm{~cm}^{-1}$ of asymmetrical stretching of the $\mathrm{CH}_{3}$ groups in HPC is observed as well as the peak at $2867 \mathrm{~cm}^{-1}$ due to the symmetric stretching band of $\mathrm{CH}_{2}$ in the cellulosic derivative [9]. At $1653 \mathrm{~cm}^{-1}$, there is a very strong peak attributed to the stretching of carbonyl bond $(\mathrm{C}=\mathrm{O})$ in PAAm, with a little peak at $1602 \mathrm{~cm}^{-1}$ which is not so strong due to the bending $\mathrm{NH}$ group of the PAAm. The absorption band at $1450 \mathrm{~cm}^{-1}$ corresponds to the asymmetric $\mathrm{CH}_{2}$ (deformation band of the PAAm and HPC). The next peak is the $1408 \mathrm{~cm}^{-1}$ attributed to $\mathrm{CH}_{2}$ out of plane symmetrical bending. The absorption band at $1271 \mathrm{~cm}^{-1}$ was assigned to a $\mathrm{CN}$ vibration of PAAm. At $1123 \mathrm{~cm}^{-1}$, there is a medium peak attributed to COC stretching and crosslinking reactions.

The spectrum of pure IBP and the gel is shown in (a) and (b) in Figure 1. The spectrum (b), in addition to the previously described bands of the gel HPC/PAAm, shows the characteristic peak of the IBP at $1719 \mathrm{~cm}^{-1}$, attributed to the stretching of the carbonyl group $\mathrm{C}=\mathrm{O}$ (typical of the carboxyl function $\mathrm{COOH}$ ) [10]. The peaks at $3080 \mathrm{~cm}^{-1}$ and $770-735 \mathrm{~cm}^{-1}$ also indicate that the structure of IBP is present

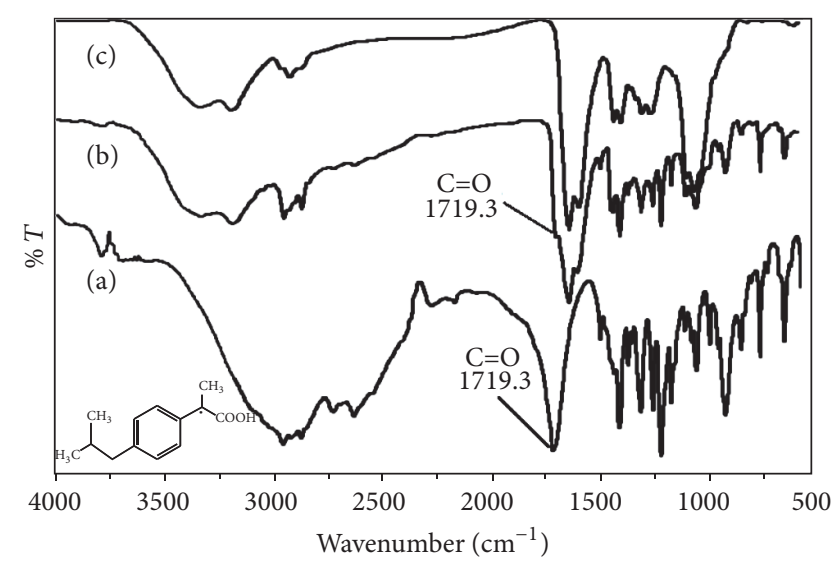

FIgURE 1: FTIR spectra of (a) IBP, (b) HPC/PAAm-IBP, and (c) HPC/PAAm.

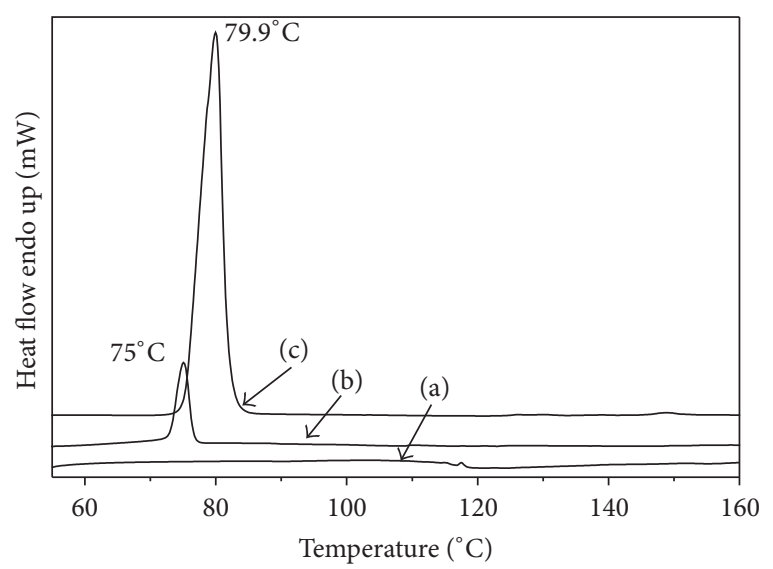

Figure 2: DSC thermograms of (a) HPC/PAAm, (b) IBP, and (c) polymer with IBP.

on the sample and that there is an orthosubstitution in the aromatic ring of IBP.

3.2. Thermal Analysis. De Brabander et al. [10] showed that ethyl cellulose compatible with IBP has a glass transition temperature $\left(T_{g}\right)$ of about 70 to $80^{\circ} \mathrm{C}$, depending on the amount of cellulose derivative. In Figure 2, the results of DSC analysis are shown. In the curve corresponding to IBP, there is a peak around $77^{\circ} \mathrm{C}$, which is characteristic of the active ingredient and corresponds to the melting point of IBP [11]. The curve of the gel with IBP has a peak at $75^{\circ} \mathrm{C}$; this shows that there is a polymer-drug interaction. When there is single $T_{g}$ in a composite of two compounds that has its own $T_{g}$, then the system is fully miscible [12]. According to the literature, at $75-76^{\circ} \mathrm{C}$ of temperature, the IBP is prismatic and irregular in shape [13].

3.3. Morphology and Structure. The yellowish appearance in the xerogel (Figure 3(a)) is conferred by PAAm and the swollen gel looks transparent, due to the water incorporated in the matrix. 


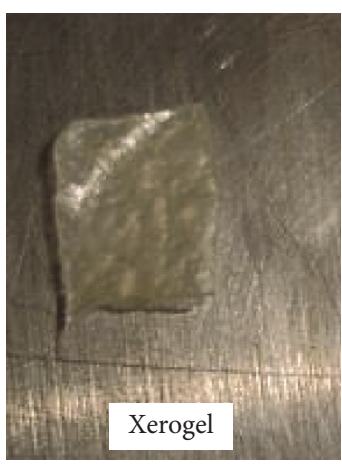

(a)

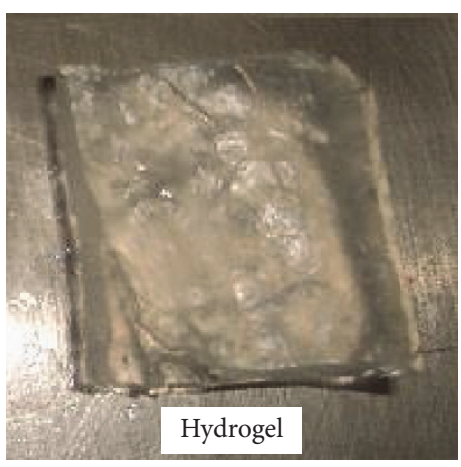

(b)

FIgURE 3: Photographs of the samples: xerogel and hydrogel.

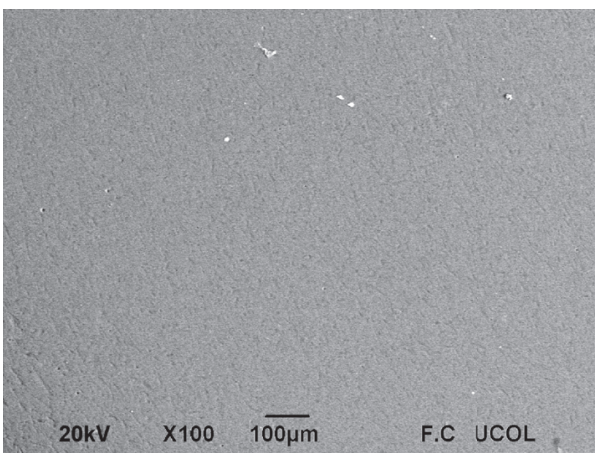

(a)

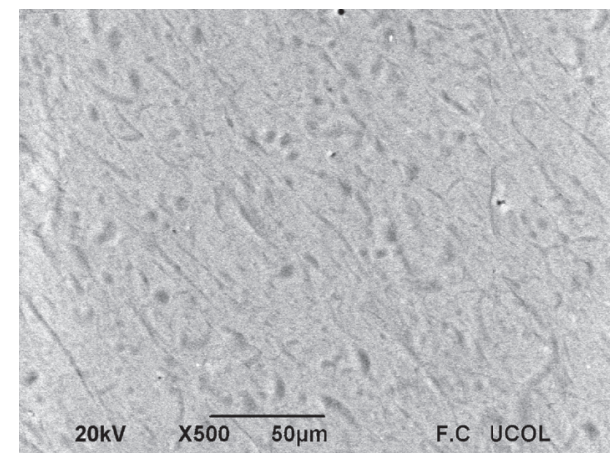

(b)

Figure 4: SEM micrographs of the HPC/PAAm xerogel surface at (a) 100x and (b) 500x.

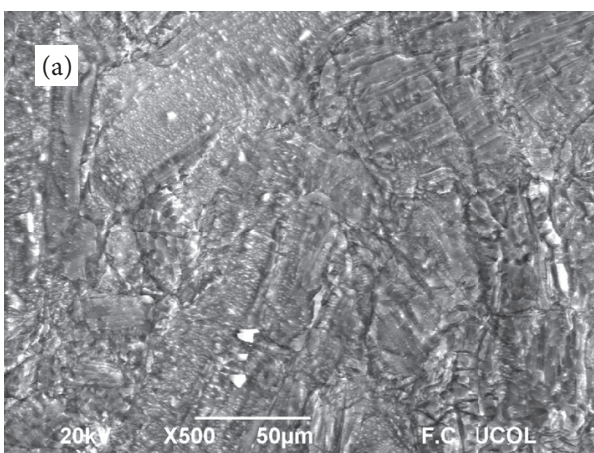

(a)

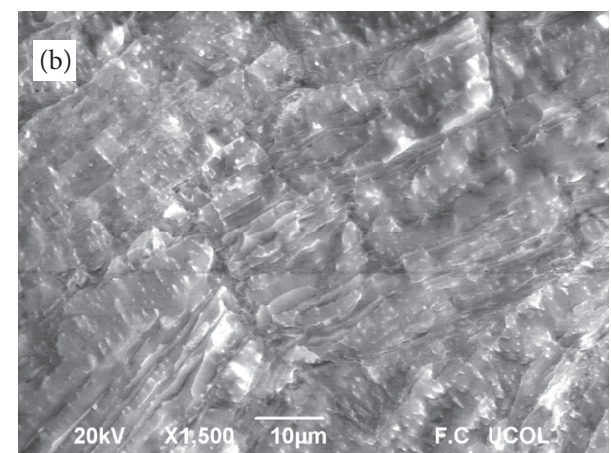

(b)

FIGURE 5: SEM micrographs of the IBP surface: (a) 500x and (b) 1500x.

In Figure 4, SEM micrographs of the sample HPC/PAAm are showed at different magnifications (100x and 500x). The pure gel has a smooth and uniform surface without the presence of agglomerates. The uniformity presented by the film indicates that there is no phase separation between the HPC and PAAm. There are pores of $6 \mu \mathrm{m}$ in diameter; the formation of channels that facilitate migration of solvent is also present.

Figure 5 corresponds to samples containing pure IBP. The images show a surface with many features, with cuts in different directions and a few shiny white spots that stand out. The sample was compressed and formed a tablet; no crystals are seen.

In Figure 6 are shown SEM micrographs at 100x and 500x of the HPC/PAAm films with IBP incorporated. A surface with rugged relief is seen in the images; this is due to the inclusion of clusters of various shapes and sizes homogeneously distributed throughout the film; these clusters correspond to crystals of IBP. This corroborates the incorporation of the drug and the existence of a drug-polymer interaction, which 


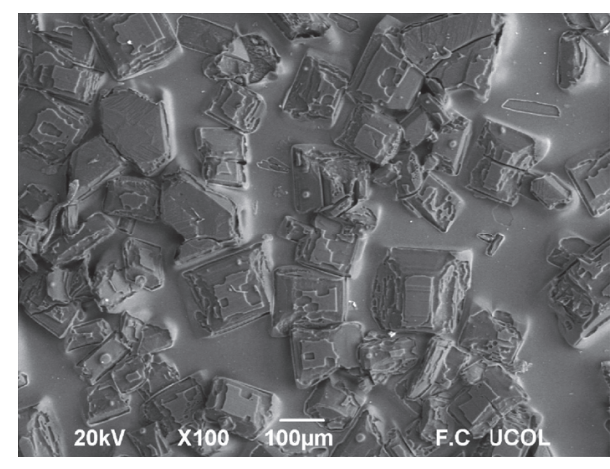

(a)

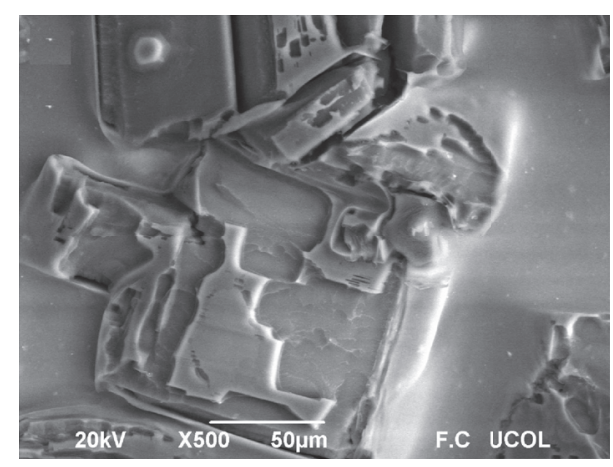

(b)

FIGURE 6: SEM micrographs of the surface of HPC/PAAm films with IBP: (a) 100x and (b) 500x.
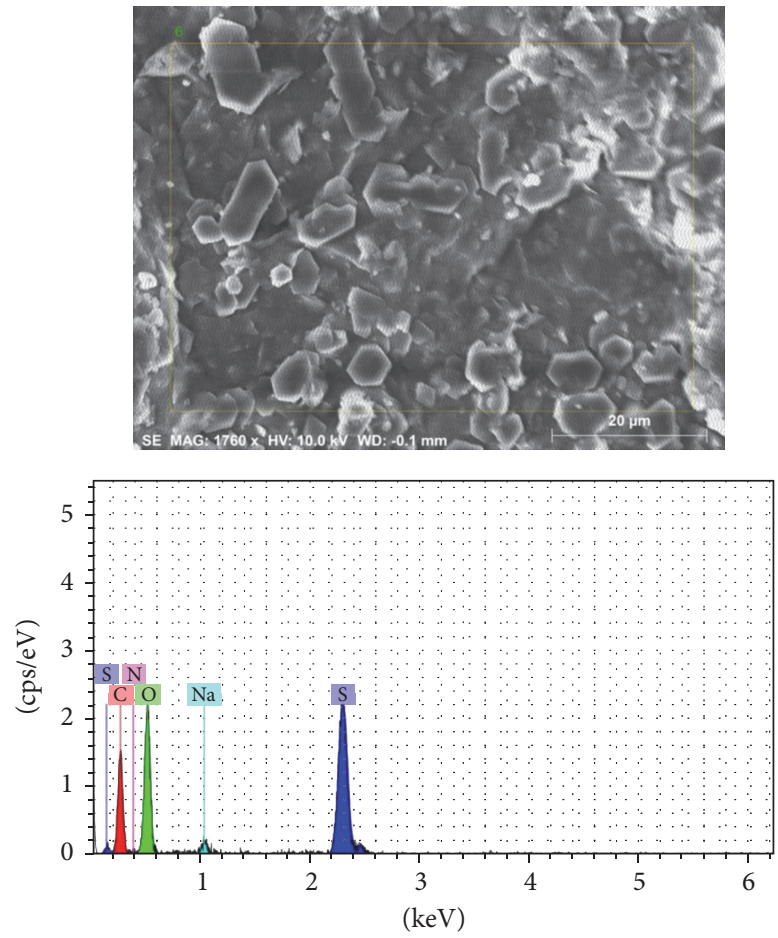

FIGURE 7: EDX analysis of the HPC/PAAm xerogel surface.

was also observed by FTIR spectra and DSC calorimetry. The clusters are shaped in some cases as a diamond with a length of $101.8 \mu \mathrm{m}$ and they grow vertically with $60 \mu \mathrm{m}$, assuming that ibuprofen is adhered to the surface in an average area of $9003 \mu \mathrm{m}^{2}$ (Figure 6(a)). Like other authors, induction to crystallization temperature is observed near $40^{\circ} \mathrm{C}$, where there is presence of growth of the crystals in the shape of circles, squares, or diamonds [14]. The time of formation of the crystals indicates that, after one hour, the crystals have sizes of $100 \mu \mathrm{m}$, and they extend from the bottom plane of the gel substrate to the top.

The HPC/PAAm gels are negative thermosensitive; because the quantity of polyacrylamide has an influence
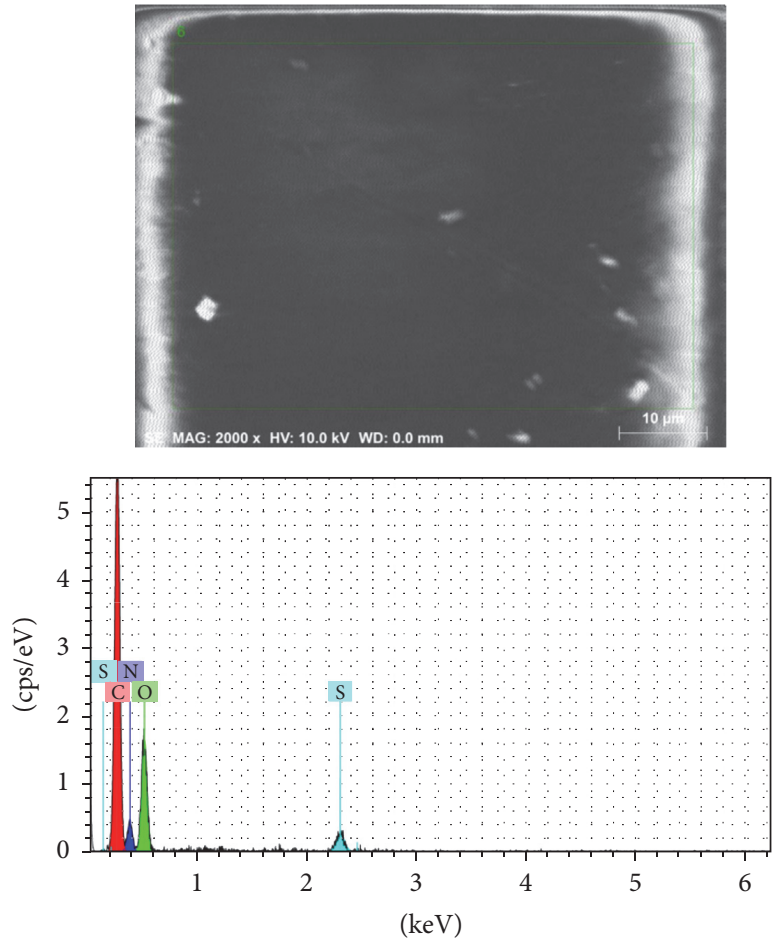

FIGURE 8: EDX analysis of the HPC/PAAm with ibuprofen, xerogel surface.

on the LCST [15-18], PAAm changes the LCST of HPC, a polymer that is known to have this property [19].

Figure 7 shows an EDX analysis of the HPC/PAAm pure polymer. The spectrum shows the presence of carbon, oxygen, sodium, and sulfur; no nitrogen was detected with the EDX. The quantitative analysis gave, in atomic percentages (at.\%), C 51.74 at.\%, O 38 at.\%, S 9.43 at.\%, and Na 0.83 at.\%. Figure 8 shows an EDX analysis performed on the polymer after absorbing ibuprofen. The analysis detected the presence of nitrogen, but no sodium was detected on the polymer. The composition of the sample with ibuprofen was C 56.92 at.\%, O 26.16 at.\%, N 16.11 at.\%, and S 0.81 at.\%. Interestingly, sulfur was detected on both samples; APS and DVS both contain 
TABLE 1: ANOVA data of mathematical models used to ascertain drug release for buffer solution.

\begin{tabular}{lccccrr}
\hline $\begin{array}{l}\text { Temperature } \\
\left({ }^{\circ} \mathrm{C}\right)\end{array}$ & \multicolumn{2}{c}{ Zero order } & \multicolumn{2}{c}{ First order } & \multicolumn{2}{c}{ Higuchi } \\
\hline 35 & $R^{2}$ & $F^{0}$ & $R^{2}$ & $F^{0}$ & $R^{2}$ & 0.00063521 \\
37 & 0.5540 & 0.005 & 0.439 & 0.0150 & 0.67534 & 0.6898 \\
39 & 0.557 & 0.00503 & 0.4312 & 0.0167 & 0.00050198 \\
\hline
\end{tabular}

TABLE 2: ANOVA data of mathematical models used to ascertain drug release for ethanol-water.

\begin{tabular}{lcccccc}
\hline $\begin{array}{l}\text { Temperature } \\
\left({ }^{\circ} \mathrm{C}\right)\end{array}$ & \multicolumn{2}{c}{ Zero order } & \multicolumn{2}{c}{ First order } & \multicolumn{2}{c}{ Higuchi } \\
\hline 35 & $R^{2}$ & $F^{0}$ & $R^{2}$ & $F^{0}$ & $R^{2}$ \\
37 & 0.6924 & $2.331 \times 10^{-24}$ & 0.3679 & $1.4299 \times 10^{-6}$ & 0.8579 & $1.2814 \times 10^{-22}$ \\
39 & 0.63585 & $6.841 \times 10^{-16}$ & 0.3998 & $7.4200 \times 10^{-9}$ & 0.80601 & $1.083 \times 10^{-24}$ \\
\hline
\end{tabular}

sulfur, so this is the source of $\mathrm{S}$ in the samples; the ratio $\mathrm{C} / \mathrm{S}$ is higher on the sample containing ibuprofen than on the sample of the polymer without the NSAID; this difference is attributed to the presence of IBP that elevated the content of carbon relative to sulfur.

3.4. Drug Release. In the PBS solution, more IBP is released than in the ethanol-water solution, about $1.17 \mathrm{mg} / \mathrm{mL}$ at $35^{\circ} \mathrm{C}$ and $0.58 \mathrm{mg} / \mathrm{mL}$ at $39^{\circ} \mathrm{C}$ in $\mathrm{PBS}$, while in ethanol-water the maximum is $0.45 \mathrm{mg} / \mathrm{mL}$ at $39^{\circ} \mathrm{C}$ and the minimum is $0.23 \mathrm{mg} / \mathrm{mL}$ at $37^{\circ} \mathrm{C}$. This suggests that the kinetics of crystallization of ibuprofen on the gel surface is preferred in PBS than in an ethanol-water solution, as various authors state that it depends on temperature, and temperatures below $40^{\circ} \mathrm{C}$ promote supersaturation of ibuprofen [14].

In order to determine the drug release kinetic model describing the dissolution profile, Matlab software was used. The simulation of the drug release of ibuprofen in buffer solution (PBS) and ethanol-water solution (EWS) was done using the mathematic models: zero, first, Higuchi, and KorsmeyerPeppas. ANOVA results are summarized in Tables 1 and 2 for zero, first, and Higuchi models and those for KorsmeyerPeppas model for both solvents are in Table 3. The F-values are not statistically significant (Tables 2 and 3 ). The confidence level is low around $80 \%$; this demonstrates that the release of IBU is not "zero" and "first order." According to the results of the kinetics of IBP, $n$ values are lower than 0.5 ; this corresponds to the ranges described by the Higuchi model, indicating the existence of several simultaneous processes in the diffusion phenomenon of ibuprofen [20].

In the first stage of this diffusional process, described by zero, first, and Higuchi, $n$ for Fickian diffusion is $>0.5$.

Cylindrical samples were used, $n=0.121$ and $K=$ 0.477 , for the Korsmeyer-Peppas model. The KorsmeyerPeppas simulation was correlated by ANOVA results for both solvents of IBU medium. From the calculated $F^{0}$, it can be inferred that the parameters and the interactions considered in the experimental design are statistically significant at $95 \%$ confidence level. The contribution of KorsmeyerPeppas simulation is shown in Figure 9; in this figure, the
TABle 3: ANOVA data of mathematical model Korsmeyer-Peppas simulation.

\begin{tabular}{lccc}
\hline Solvent & Temperature $\left({ }^{\circ} \mathrm{C}\right)$ & $R^{2}$ & $F^{0}$ \\
\hline Buffer & 35 & 0.86906 & 0.000052 \\
Buffer & 37 & 0.93486 & 0.00000314 \\
Buffer & 39 & 0.94927 & 0.00000115 \\
\hline Ethanol-water & 35 & 0.79316 & $1.3162 \times 10^{-18}$ \\
Ethanol-water & 37 & 0.823801 & $4.9406 \times 10^{-26}$ \\
Ethanol-water & 39 & 0.745548 & $6.6297 \times 10^{-21}$ \\
\hline
\end{tabular}

Korsmeyer-Peppas contribution is shown as an isotherm. It can be observed from Table 3 that the parameter $F$ is the most significant factor with $37^{\circ} \mathrm{C}$ for both solvents. The best solvent to release the IBP is the buffer solution. Using the simulation, the release of ibuprofen at the first 50 minutes is clearly demonstrated. The mathematical model of Korsmeyer-Peppas is the best correlation between the minimum diffusional exponents.

If we try to correlate the observations from the SEM micrographs, where IBP clusters are seen, there is a threshold of dispersed percolation, and the critical percolation probability is preferred at $39^{\circ} \mathrm{C}$ in an ethanol-water solution. The buffer solution releases IBP in smaller quantities without diffusion restriction by polymeric chain relaxation or erosion.

3.5. XRD. Figure 10 shows the powder diffractograms of the pure polymer and of the polymer with IBP. The result for pure HPC/PAAm, shown in Figure 10(a), is very similar to HPC with a molar substitution (MS) of 4 [21], with reflections of $2 \theta$ at $7^{\circ}$ and $21^{\circ}$; the material of the diffractogram has a low degree of crystallinity, as it shows an amorphous halo diffraction pattern [22], and the sample is noisy, with the peak at $40^{\circ}$ being broad and having noise. The diffractogram of the HPC/PAAm gel is shown in Figure 10(b); the intensity of the peak at $21^{\circ}$ is increased and the sample looks less amorphous; however, the peaks of IBP are not seen on the diffractogram, and this indicates that there is a reduction in crystallinity or 


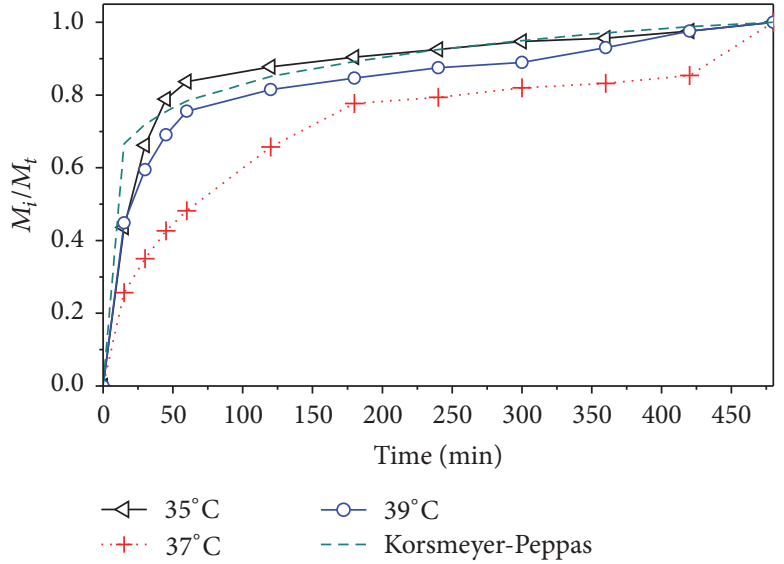

(a)

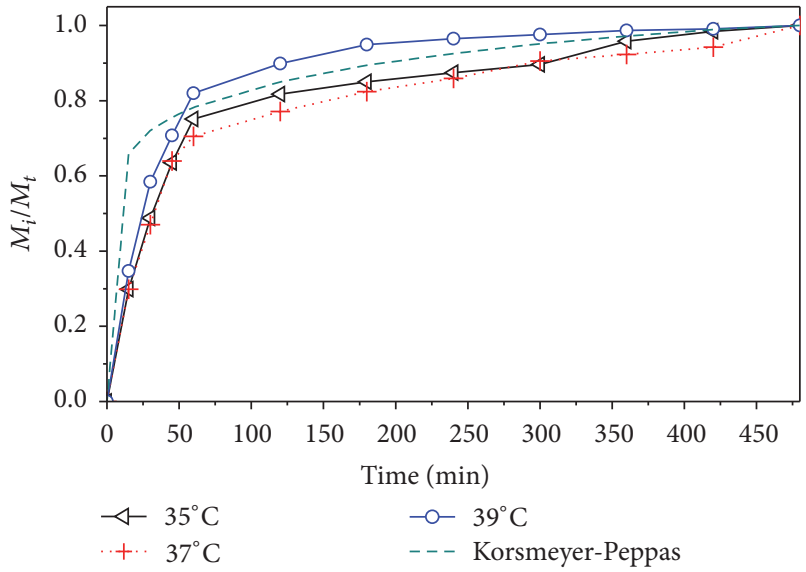

(b)

FIGURE 9: Release of IBP using (a) buffer solution and (b) ethanol-water.

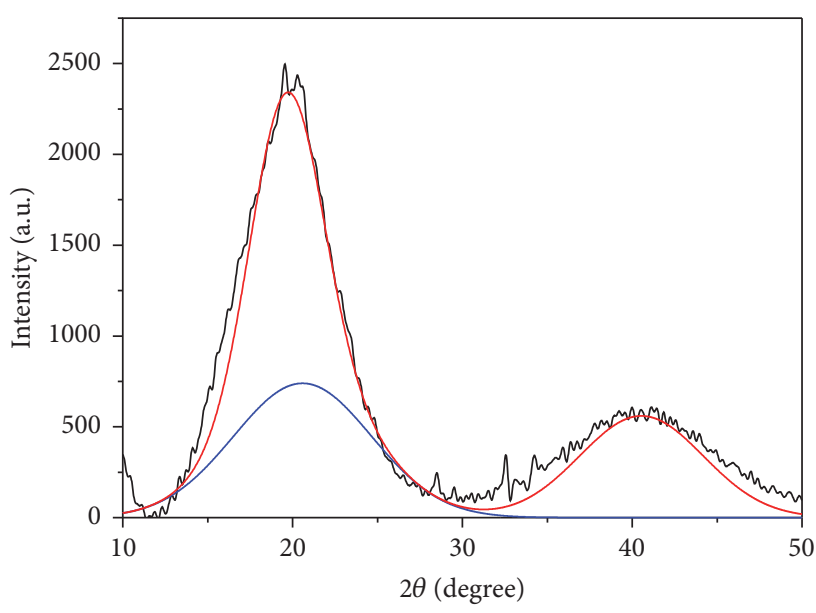

(a)

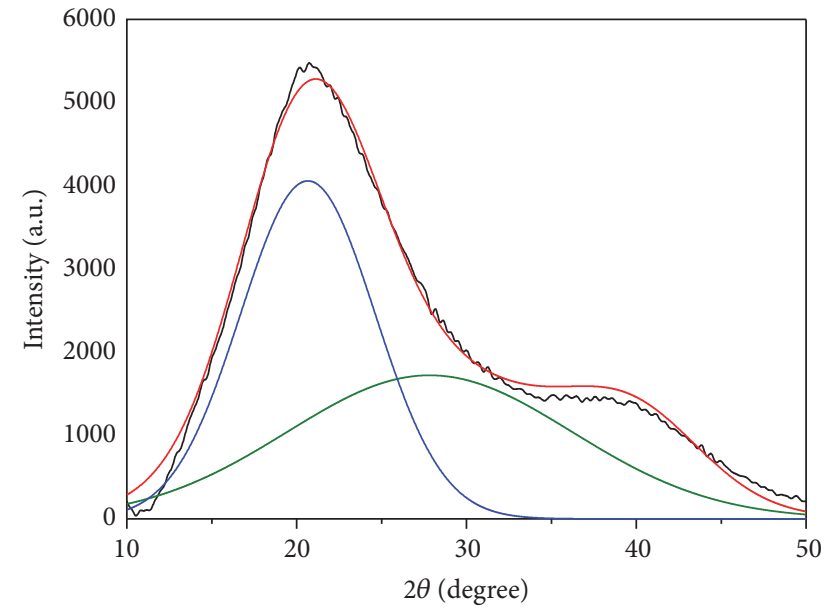

(b)

FIGURE 10: Powder diffractograms of (a) HPC/PAAm and (b) HPC/PAAm with IBP.

a change in crystal size because there is a higher quantity of polymers [4, 23-25].

The crystallization grades of the samples were calculated for the HPC/PAAm gel, having $68 \%$ of crystallinity, and the sample HPC/PAAm-IBP has 58\% of crystallinity; the results are according to reports in literature $[23,24]$.

\section{Conclusions}

IBP was incorporated into HPC/PAAm films and the release kinetics was measured. The incorporation of the NSAID was verified with FTIR. The spectra showed that the IBP peaks are present on the films after the incorporation of the drug; this observation was confirmed with the DSC thermograms, which showed a peak at $75-77^{\circ} \mathrm{C}$ consistent with the presence of IBP on the polymer. The peak showed by DSC suggested that IBP crystals had a prismatic shape [13] and that there is a polymer-drug interaction. The SEM micrographs showed the presence of IBP crystals on the surface of the polymer, corroborating the results of FTIR and DSC. EDX indicated a change in the ratio $\mathrm{C} / \mathrm{S}$ in the samples, suggesting the incorporation of a molecule having carbon and no sulfur, as the molecule of ibuprofen. There is a change in the size of the crystals of IBP during the process of incorporation of the NSAID to the polymer, as indicated by XRD.

The HPC/PAAm gel is able to release the IBP that was incorporated in the polymer. The polymer was able to release $1.1 \mathrm{mg} / \mathrm{mL}$ of IBP at $35^{\circ} \mathrm{C}$ in PBS; the kinetics of the drug release is a non-Fickian phenomenon. In this case, the NSAID is released by a diffusion phenomenon and by viscoelastic relaxation of the polymer during the simultaneous swelling process [1].

\section{Competing Interests}

The authors declare that there is no conflict of interests regarding the publication of this article. 


\section{Acknowledgments}

C. Castillo-Miranda and H. Velasco-Ocejo would like to thank SEP and CONACYT (no. 572436) for the scholarship for graduate studies. The authors are grateful to Mr. D. Pozas Zepeda (University of Colima, Faculty of Science) and R. Morán (National Autonomous University of Mexico, Campus IER Cuernavaca) for the SEM images. The article was published with the support of PRODEP.

\section{References}

[1] A. K. Bajpai, S. K. Shukla, S. Bhanu, and S. Kankane, "Responsive polymers in controlled drug delivery," Progress in Polymer Science, vol. 33, no. 11, pp. 1088-1118, 2008.

[2] X. Chen, S. Wang, M. Lu et al., "Formation and characterization of light-responsive TEMPO-oxidized konjac glucomannan microspheres," Biomacromolecules, vol. 15, no. 6, pp. 2166-2171, 2014.

[3] C. Castro, A. Morales, A. Koschella, and T. Heinze, "Phase behaviour of hydroxypropyl cellulose/polyacrylamide gels," Macromolecular Symposia, vol. 296, no. 1, pp. 429-435, 2010.

[4] J. Siepmann and N. A. Peppas, "Modeling of drug release from delivery systems based on hydroxypropyl methylcellulose (HPMC)," Advanced Drug Delivery Reviews, vol. 48, no. 2-3, pp. 139-157, 2001.

[5] M. V. Risbud, A. A. Hardikar, S. V. Bhat, and R. R. Bhonde, "pHsensitive freeze-dried chitosan-polyvinyl pyrrolidone hydrogels as controlled release system for antibiotic delivery," Journal of Controlled Release, vol. 68, no. 1, pp. 23-30, 2000.

[6] T. J. Higuchi, "Mechanism of sustained-action medication. Theoretical analysis of rate of release of solid drugs dispersed in solid matrices," Journal of Pharmaceutical Sciences, vol. 52, no. 12, pp. 1145-1149, 1963.

[7] R. W. Korsmeyer and N. A. Peppas, "Macromolecular and modeling aspects of swelling-controlled systems," in Controlled Release Delivery Systems, T. J. Roseman and S. Z. Mansdorf, Eds., pp. 77-90, Dekker, New York, NY, USA, 1983.

[8] J. Mark, Polymer Data Handbook, Oxford University Press, New York, NY, USA, 1999.

[9] C. F. Castro-Guerrero and D. G. Gray, "Chiral nematic phase formation by aqueous suspensions of cellulose nanocrystals prepared by oxidation with ammonium persulfate," Cellulose, vol. 21, no. 4, pp. 2567-2577, 2014.

[10] C. De Brabander, G. Van Den Mooter, C. Vervaet, and J. P. Remon, "Characterization of ibuprofen as a nontraditional plasticizer of ethyl cellulose," Journal of Pharmaceutical Sciences, vol. 91, no. 7, pp. 1678-1685, 2002.

[11] J. D. Higgins, T. P. Gilmor, S. A. Martellucci, R. D. Bruce, and H. G. Brittain, "Ibuprofen," in Analytical Profiles of Drug Substances and Excipients, H. G. Brittain, Ed., vol. 27, pp. 265300, Academic Press, New York, NY, USA, 2001.

[12] R. J. Babu, W. Brostow, I. M. Kalogeras, and S. Sathigari, "Glass transitions in binary drug + polymer systems," Materials Letters, vol. 63, no. 30, pp. 2666-2668, 2009.

[13] G. M. Khan and Z. Jiabi, "Preparation, characterization, and evaluation of physicochemical properties of different crystalline forms of ibuprofen," Drug Development and Industrial Pharmacy, vol. 24, no. 5, pp. 463-471, 1998.

[14] A. Rashid, E. T. White, T. Howes, J. D. Litster, and I. Marziano, "Growth rates of ibuprofen crystals grown from ethanol and aqueous ethanol," Chemical Engineering Research and Design, vol. 90, no. 1, pp. 158-161, 2012.

[15] J. Heiko, L. Vander, H. Sebastiaan, O. Wonter, and B. Piet, "Stimulus-sensitive hydrogels and their application in chemical (micro) analysis," The Royal Society of Chemistry, vol. 128, pp. 325-331, 2003.

[16] A. S. Hoffman, P. S. Stayton, V. Bulmus et al., "Really smart bioconjugates of smart polymers and receptor proteins," Journal of Biomedical Materials Research, vol. 52, no. 4, pp. 577-586, 2000.

[17] Y. Qiu and K. Park, "Environment-sensitive hydrogels for drug delivery," Advanced Drug Delivery Reviews, vol. 53, no. 3, pp. 321-339, 2001.

[18] S. H. Cho, M. S. Jhon, S. H. Yuk, and H. B. Lee, “Temperatureinduced phase transition of poly $(\mathrm{N}, \mathrm{N}$-dimethylaminoethyl methacrylate-co-acrylamide)," Journal of Polymer Science, Part B: Polymer Physics, vol. 35, no. 4, pp. 595-598, 1997.

[19] R. S. Werbowyj and D. G. Gray, "Ordered phase formation in concentrated hydroxypropylcellulose solutions," Macromolecules, vol. 13, no. 1, pp. 69-73, 1980.

[20] D. R. Lide, Handbook of Chemistry and Physics, CRC, Boca Raton, Fla, USA, 76th edition, 1996.

[21] R. J. Samuels, "Solid-state characterization of the structure and deformation behavior of water-soluble hydroxypropylcellulose," Journal of Polymer Science Part A, vol. 7, no. 7, pp. 1197$1258,1969$.

[22] R. Talukder, C. Reed, T. Dürig, and M. Hussain, "Dissolution and solid-state characterization of poorly water-soluble drugs in the presence of a hydrophilic carrier," AAPS PharmSciTech, vol. 12, no. 4, pp. 1227-1233, 2011.

[23] M. Nagpal, S. K. Singh, and D. Mishra, "Superporous hybrid hydrogels based on polyacrylamide and chitosan: characterization and in vitro drug release," International Journal of Pharmaceutical Investigation, vol. 3, no. 2, pp. 88-94, 2013.

[24] S. V. Dalvi and R. N. Dave, "Analysis of nucleation kinetics of poorly water-soluble drugs in presence of ultrasound and hydroxypropyl methyl cellulose during antisolvent precipitation," International Journal of Pharmaceutics, vol. 387, no. 1-2, pp. 172-179, 2010.

[25] L. Segal, I. J. Creely, A. E. Martin Jr., and C. M. Conrad, "An empirical method for estimating the degree of crystallinity of native cellulose using the X-ray diffractometer," Textile Research Journal, vol. 29, no. 10, pp. 786-794, 1959. 

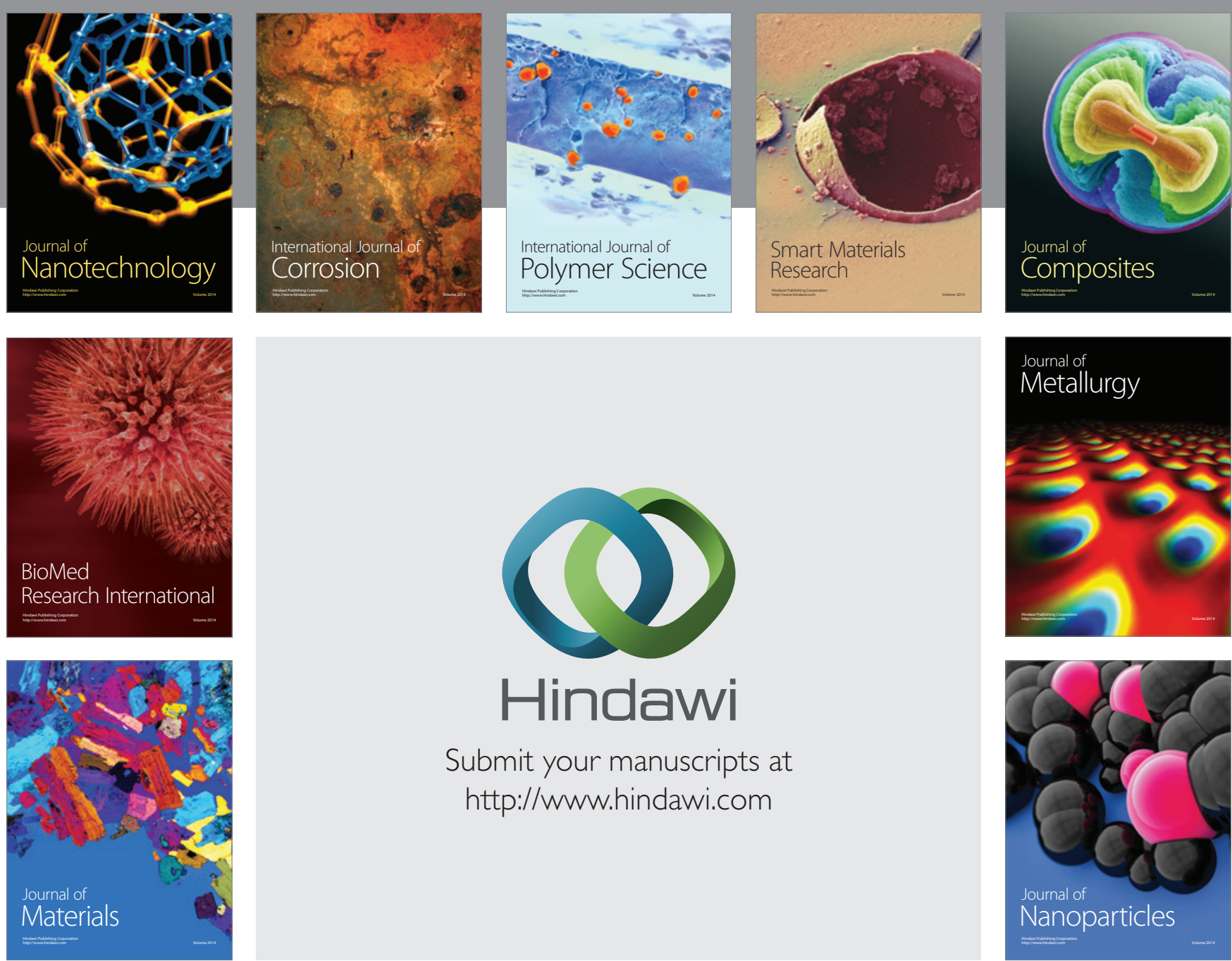

\section{Hindawi}

Submit your manuscripts at

http://www.hindawi.com

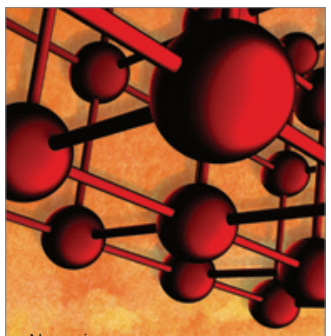

Materials Science and Engineering
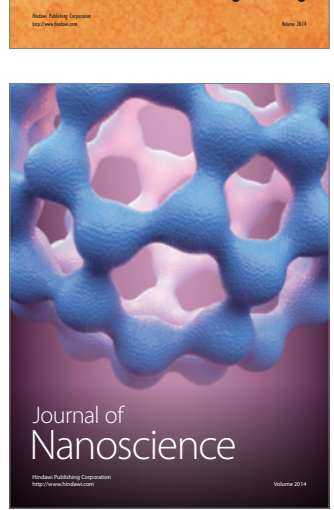
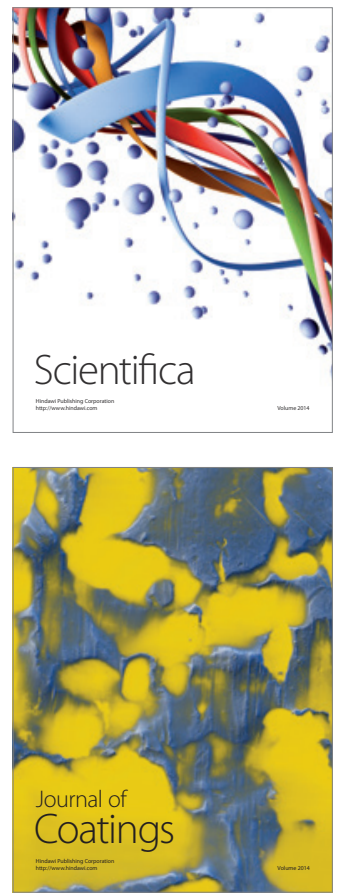
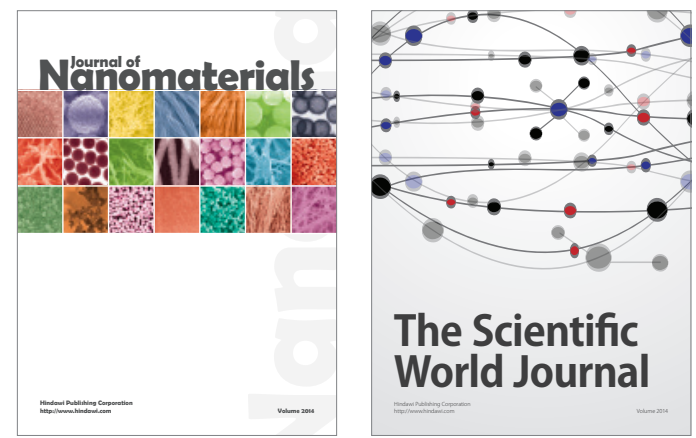

The Scientific World Journal
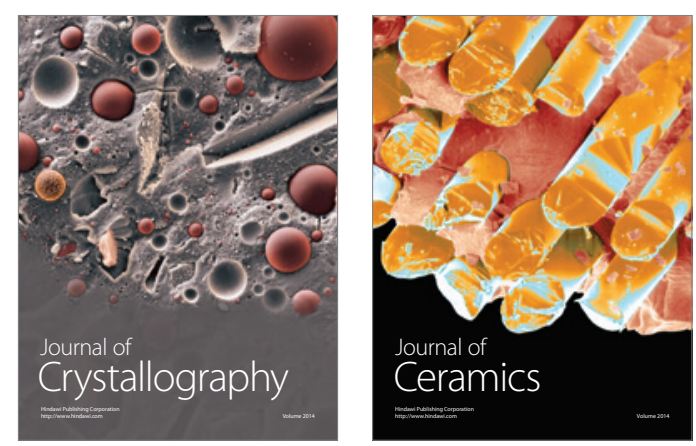
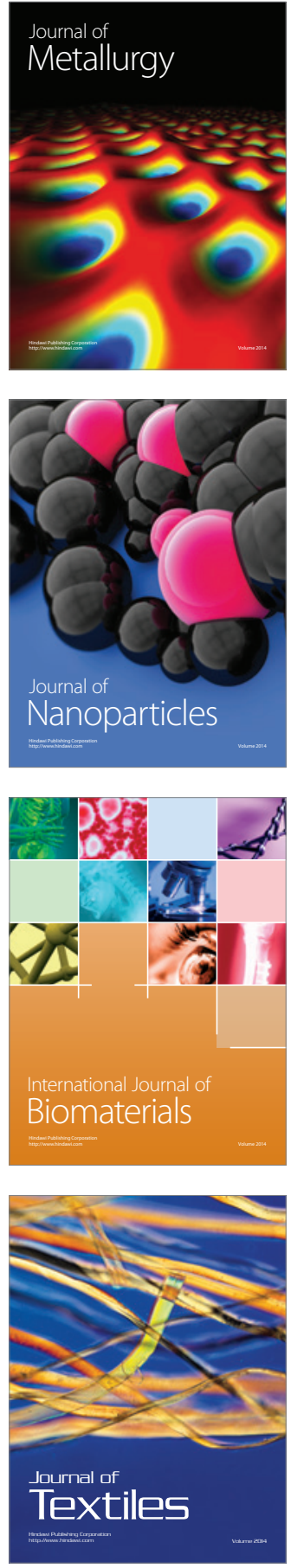